\title{
PROKLA-Redaktion
}

\section{Editorial: Arbeit und Wertschöpfung im digitalen Kapitalismus}

Die Diskussion um die Zukunft der Arbeit wird derzeit von Spekulationen über die Auswirkungen der Digitalisierung überlagert. In Deutschland konzentriert sich die Debatte, unterfüttert von üppig ausgestatteten Förderprogrammen, um den Begriff „Industrie 4.0“. Dies hat zunächst einmal den Effekt, dass die Entwicklung der Produktivkräfte und speziell der Industriearbeit plötzlich wieder en vogue sind. Das ist durchaus zu begrüßen, wurden die materiellen Grundlagen wirtschaftlichen Handelns doch seit den 1990er Jahren durch übertriebene Prognosen zur New Economy in den Hintergrund gedrängt (vgl. hierzu die Diskussion in PROKLA 122). Die Fiktion einer "Entstofflichung" der Ökonomie (Albert u.a. 1999) hat heute einem neuen Interesse an Arbeit und Produktion Platz gemacht. Damit erhält der deutsche Digitalisierungsdiskurs eine markant andere und weniger verklärende Note als jener im angloamerikanischen Raum, wo das Ende der Arbeit schon eine ausgemachte Sache zu sein scheint (vgl. Mason 2016; Srnicek/Williams 2015).

Zugleich schafft der Begriff „Industrie 4.0" neue Mythen. Zum einen, weil er sich in geradezu bornierter Weise auf die Kernbereiche der deutschen Exportindustrie beschränkt und damit wichtige Dimensionen der Digitalisierung von Arbeit aus dem Blickfeld verliert. Zum anderen, weil „Industrie 4.0" eine politische Agenda verkörpert, ja, als ein neues Hegemonieprojekt der Unternehmensverbände und staatlichen Eliten verstanden werden kann. So erscheint der Begriff „Industrie 4.0“ wie eine Zauberformel, mit der das Ende der stagnierenden Konjunkturentwicklung beschworen wird. Der Branchenverband BITKOM rechnet bis 2025 mit einem sagenhaften Wachstumsplus von 1,7 Prozent pro Jahr infolge von Produktivitätsgewinnen in der „Industrie 4.0“ (BITKOM/Fraunhofer IAO 2014: 35). Mit solch rosigen Aussichten auf eine Digitalisierungsdividende und mit einer neuen sozialpartnerschaftlichen Perspektive, nach der der Mensch im Mittelpunkt digitalisierter Produktionssysteme stehe, ist das Projekt „Digitalisierung“ eine zentrale Legitimitätsressource der Machteliten in Unternehmen, Staat und Wissenschaft. Der Diskurs wirkt wie eine offensive Antwort auf eine Defensive in der öffentlichen Diskussion: Weil rasches Wachstum in der gegenwärtigen ökonomischen Stagnationsperiode westlicher Gesellschaften nicht mehr herzustellen ist und die soziale Ungleichheit zunimmt (Nachtwey 2016: 15ff.), setzen die Herrschenden auf ein Heilsversprechen in der Zukunft. Die Vision einer neuen industriellen Revolution, in deren Folge nichts mehr so ist wie zuvor, eignet sich für Projektionen verschiedenster Art - von der sozialpartnerschaftlichen Utopie bis zur Dystopie einer umfassenden Vollautomatisierung und der Herrschaft künstlicher Intelligenz über die Menschen (vgl. Butollo/Engel 2015). 
Diese diskursive Konstellation stellt die kritische Sozialwissenschaft vor besondere Herausforderungen. Die Mythen des offenkundig interessengeleiteten Begriffs „Industrie 4.0“ müssen hinterfragt werden, während mögliche Umbrüche nicht übersehen werden dürfen, indem sie schlicht als neuer Aufwasch alter Diskussionen abgetan werden. Es reicht beispielsweise nicht zu behaupten, dass die menschenleere Fabrik nicht kommen werde, wenn neue Formen der Arbeitsorganisation, der Automation und der Kontrolle von Arbeit Einzug halten. Die Einsicht darüber, dass mancher Medienbericht über die Substitution lebendiger Arbeit übertrieben ist und dass zwischen technischer Machbarkeit und tatsächlicher Implementierung Welten liegen, sollte nicht zu Behäbigkeit gegenüber tatsächlich einsetzenden Veränderungen führen. Denn auch wenn der Technologieeinsatz keinen eindeutig bestimmenden Einfluss auf die Gestaltung der Arbeit hat, so bedarf es doch konkreter Analysen der jeweiligen technischen Systeme, um Veränderungen nachzeichnen und Konfliktfelder umreißen zu können (Pfeiffer 2010). Die Frage danach, was alt ist und was neu, wo Kontinuitätslinien zu verzeichnen sind und wo Brüche in der Organisation von Wertschöpfung und Arbeit liegen, durchzieht daher die Beiträge in dieser Ausgabe.

Statt jedoch über zukünftige Entwicklungen zu rätseln, nehmen die Autor_innen dieser Schwerpunktausgabe eine sachliche Bestandaufnahme der Widersprüche neuer sozio-technischer Entwicklungen vor. Wer sich nur an den Vorstellungen über die Mensch-Maschine-Interaktion in der $\mathrm{Zu}$ kunft orientiert, abstrahiert von konkreten Kräfteverhältnissen, die über die Umsetzung und auch darüber entscheidet, wer bei der Digitalisierung gewinnt und wer verliert. Technologien determinieren keine gesellschaftlichen Prozesse, wie es viele Analysen und Konzepte zur „Industrie 4.0" mit ihrer Stufenabfolge von technologischen Revolutionen suggerieren (BMBF 2013). Wohl aber können durch Technologien Kräfteverhältnisse konsolidiert und verschoben werden. Dies ist sicherlich keine neue Erkenntnis, schließlich ging die mikroelektronische Revolution mit dem Aufkommen des Neoliberalismus und der Globalisierung einher. Emanzipatorische Potenziale neuer Technologien konnten sich kaum durchsetzen, weil für ihren Einsatz andere Interessen ausschlaggebend waren. Die starke Kopplung technologischer Investitionen an die öffentliche Förderung und die Kapitalmärkte stellt sicher, dass Dividenden privatisiert werden - „Surfen auf der Innovationswelle“ nennt Mazzucato (2014) dieses Problem. Darum ist auch zu befürchten, dass Tendenzen der Prekarisierung und der sozialen Spaltung die Anwendung der kommenden Phasen digitaler Technologienutzung prägen. Dies rechtfertigt einen kritischen Zugang zum Thema, den die Prokla mit dieser Ausgabe gewählt hat.

Diese Kritik kann als Orientierung für politisches Handeln dienen. Als „Einsicht in die Notwendigkeit“ geht es darum, Kräfteverhältnisse zu vermessen, um Handlungsmöglichkeiten auszuloten. Angesichts der Wirkmächtigkeit des technologischen Wandels reicht es nicht aus, pauschal dessen Gestaltbarkeit zu postulieren. Aus einer Kritik an der Verdinglichung gesellschaftlicher Zustände wird schnell blinder Gestaltungsoptimismus, wenn die Analyse gesellschaftlicher Kräfteverhältnisse ausbleibt (vgl. Urban 2016). In diesem Sinne verweisen die Beiträge dieser Ausgabe darauf, dass der Technologieeinsatz nur selten demokratisch verhandelt wird und die Interessen von Unternehmen und Finanzmarktakteuren in der Regel eine 
höhere Wirkmächtigkeit entfalten als die Interessen der Subalternen.

Ein erster Teil des Themenschwerpunkts bezieht sich auf die Frage nach neuen Formen der Kontrolle und der Transformation von Arbeit im digitalisierten Kapitalismus. Moritz Altenried gibt zunächst einen Überblick über den neu entstandenen Jedermanns-Arbeitsmarkt der digitalen Click- und Crowdworker. Er analysiert, wie die tayloristische Zerlegung und Vergabe von Tätigkeiten durch Online-Plattformen als einer Art digitale Infrastruktur der Produktion funktioniert, in deren algorithmische Architektur wiederum Klassenverhältnisse eingeschrieben sind. Auch wenn auf Abruf verfügbare Clickworker letztlich in den Randbereichen des Arbeitsmarkts aktiv sind und oftmals nur ein Zubrot zum Haupterwerb verdienen, stellt sich im globalen Plattformkapitalismus die Frage nach der Existenzsicherung durch Erwerbsarbeit dennoch neu. Der Beitrag öffnet den Blick für die universelle Mobilisierung bisher unerschlossener Arbeitskraftressourcen über Ländergrenzen hinweg.

Doch nicht nur in der digitalen Welt der Onlineplattformen wird Einfacharbeit geschaffen und entstehen neue Formen von Prekarität. Auch in der analogen Welt, etwa der deutschen Industrie, hat die Digitalisierung der Arbeit Prekarisierungsprozesse in Gang gesetzt. So identifizieren Martin Ehrlich, Thomas Engel, Walid Ibrahim und Manfred Füchtenkötter in ihrer empirischen Untersuchung klassischer Industriebranchen zwar eine qualifikatorische Aufwertung einzelner Beschäftigtengruppen, aber auch neue Treiber der Prekarisierung und psychischer Belastungen. Ihre Fallstudien deuten auf eine erhöhte soziale Verwundbarkeit von Industriebeschäftigten durch die Einführung neuer digitalen Technologien hin.
Eine ähnlich skeptische Position beziehen Jobst Gaus, Christopher Knop und David Wandjo. Sie zeigen auf Basis empirischer Studien, dass die individualisierte Fertigung nach Kundenwunsch, die einen Kern des Industrie 4.0-Paradigmas darstellt, innerbetriebliche Prozesse unmittelbar an die Marktanforderungen bindet. Der optimistischen Prognose der Ausweitung von Dispositionsspielräumen bis hin zu einer Demokratisierung von Unternehmen stellen sie ihre Diagnose eines Ablaufdeterminismus entgegen. Der Kundenwunsch diktiert mehr und mehr die Rhythmen der Herstellung, dem sich die Beschäftigten fügen müssen. Bestehende Formen der Mitbestimmung und Partizipation von Beschäftigten wird auf diese Weise zu einer abhängigen Variable der Ablaufoptimierung und verliert an Substanz.

Auch Eva-Maria Raffetseder, Simon Schaupp und Philipp Staab geht es in ihrem Beitrag um die Frage nach neuen Formen betrieblicher Kontrolle von Arbeit, die Perspektive der AutorInnen fokussiert dabei aber explizit auf neue Steuerungssoftware und deren Folgen für Arbeit. Sie untersuchen auf empirischer Basis die Steuerungssoftware Salesforce und sensorenbasierte Feedbacksysteme. Solche Formen kybernetischer Kontrolle durch algorithmische Steuerung kommen aufgrund ihres reflexiven Charakters ohne kognitive Planung und hierarchische Ordnung aus, werden von den AutorInnen aber dennoch als neue Qualität von Herrschaft im digitalen Kapitalismus interpretiert.

Die neuen Formen von Arbeit und die verschärfte Ausbeutung der Ware Arbeitskraft im digitalen Kapitalismus führen jedoch auch zu Gegenwehr und Alternativentwürfen. Diesen neuen Konflikten ist der zweite Teil der Schwerpunktausgabe gewidmet. Georg Barthel und Jan 
Rottenbach zeichnen nicht nur die neuen Methoden digitalisierter Kontrolle beim E-Commerce Giganten Amazon nach, sondern präsentieren auch Befunde über widerständige Strategien und Praktiken in der „panoptischen Fabrik“. Ihre Studie, die auf einer Mit-Untersuchung nach operaistischem Vorbild beruht, arbeitet neben den Organisierungsprozessen die verdeckten Formen des Arbeitskonflikts bei Amazon heraus. Solche Mikrokonflikte wenden sich gegen das strikte Arbeitsregime in der Dienstleistungsfabrik und könnten perspektivisch größere Kollektivaktionen ermöglichen.

Auch in anderen Bereichen des digitalen Kapitalismus ist es zu Arbeitskonflikten gekommen. Am Beispiel des Foodora-Streiks in Turin analysieren Stefania Animento, Giorgio Di Cesare und Cristian Sica neue Konfliktlinien in der gig economy. Dabei beschreibt die Gruppe, wie die riders bei Foodora sich kollektiv organisierten, um ihre Forderungen für bessere Bezahlung und Arbeitsbedingungen Nachdruck zu verleihen. In der Auseinandersetzung wurden neue Formen des Arbeitskampfes erprobt: Auf die digitale Aussperrung durch die Arbeitgeber aus der App antworteten die Protestierenden mit einem Shitstorm gegen die Onlinepräsenz des Unternehmens. Auch wenn durch den Protest nur ein Teilerfolg erzielt wurde, trug dieser dazu bei, dass in Italien eine öffentliche Debatte über die Arbeitsbeziehungen in der gig economy begann.

Mit dem Beitrag von Florian Butollo und Yannick Kalff zu den Thesen von Paul Mason schließt der thematische Schwerpunkt zu digitaler Arbeit und Wertschöpfung. Mason geht davon aus, dass die Eigentumsverhältnisse im digitalen Kapitalismus zunehmend brüchig werden und sich ein langsamer, politisch moderierter Übergang zum Postkapitalismus auf Kooperationsformen jenseits des Marktes, die sogenannte sharing economy, stützen könne. Butollo und Kalff halten dem entgegen, dass Akte des Teilens in kapitalistische (Re-)Produktions- und Klassenverhältnisse eingebunden bleiben und Unternehmen neue Wege entwickelt haben, vermeintlich freie Kooperationen im Internet in Wert zu setzen. Ihre Kritik an Paul Masons Buch versteht sich als Beitrag zur Strategiedebatte, der ein allgemeines Dilemma anspricht: das Verhältnis des Kampfes um digitale (und analoge) Freiräume im Hier und Jetzt und deren Verhältnis zu einer Systemtransformation.

Zusammengefasst: Wir sind längst in der Wirklichkeit der vermeintlichen Digitalisierungsrevolution angekommen, Arbeit und Wertschöpfung im digitalen Kapitalismus weisen Schattenseiten auf, die sich in Prekarisierung, Kontrolle und wachsenden Belastungen am Arbeitsplatz äußern. Doch beinahe so schnell, wie sich der digitale Wandel vollzogen hat, hat sich bereits Widerstand formiert.

Außerhalb des Schwerpunkts zeichnet Christian Christen nach, wie sich der neoliberale Rentenkonsens in Deutschland entwickelt hat. Die "Reformen“ des Alterssicherungssystems der letzten 20 Jahre war Teil eines internationalen Transformationsprozesses. Kernstück ist die Umstellung auf kapitalgedeckte Modelle und die individuelle Vorsorge. Die Versprechen wurden nicht eingelöst und vielen Menschen droht die Altersarmut. Dennoch herrscht ein stabiler Rentenkonsens, obwohl eine grundlegende Revision überfällig ist - nicht zuletzt, um einen möglichen Zusammenbruch des Rentensystems in den nächsten Jahrzehnten abzuwenden.

Ewgeniy Kasakow führt unsere Reihe zur Russischen Revolution fort. In den linken Debatten gehöre die weitverbreitete 
Vorstellung, 1917 wären in Russland ArbeiterInnen und BäuerInnen im Begriff gewesen, ihre eigene Vorstellung von Sozialismus zu realisieren und lediglich die bolschewistische Bürokratie hätte sie daran gehindert. Die Räte gelten vielen Linken als eine Alternative zu den autoritären Bolschewiki. Nur selten werden jedoch solche Annahmen anhand Archivalien und neuen Studien überprüft.

\section{Florian Butollo, Thomas Engel und Stefan Schmalz (für die Redaktion)}

Als Gastredakteure haben Florian Butollo und Thomas Engel am Schwerpunkt dieses Heftes mitgewirkt. Die PROKLARedaktion dankt ihnen ganz herzlich für ihre Ideen und ihr Engagement.

Anfang Mai 2017 fand die Mitgliederversammlung der Vereinigung zur Kritik der politischen Ökonomie e.V. statt, der die PROKLA herausgibt und die Redaktion und den Redaktionsbeirat wählt. Die Redaktion wird durch Stefanie Graefe und Felix Syrovatka gestärkt - wir freuen uns auf die Zusammenarbeit.

\section{Literatur}

Albert, Mathias/Brock, Lothar/Hessler, Stephan/Menzel, Ulrich und Neyer, Jürgen (1999): Die neue Weltwirtschaft. Entstofflichung und Entgrenzung der Ökonomie. Frankfurt/M.

BITKOM/Fraunhofer IAO (2014): Industrie 4.0 - Volkswirtschaftliche Potentiale für Deutschland. Berlin-Stuttgart.

BMBF (2013): Deutschlands Zukunft als Produktionsstandort sichern. Umsetzungsempfehlungen für das Zukunftsprojekt Industrie 4.0 - Abschlussbericht des Arbeitskreises Industrie 4.0. Berlin.

Butollo, Florian/Engel, Thomas (2015): Industrie 4.0 -- arbeits- und gesellschaftspolitische Perspektiven. Zwischen Dystopie und Euphorie. In: Z. Zeitschrift für Marxistische Erneuerung 26(103), 29-41.

Mason, Paul (2016): Postkapitalismus. Grundrisse einer kommenden Ökonomie. Berlin.

Mazzucato, Mariana (2014): Das Kapital des Staates. Eine andere Geschichte von Innovation und Wachstum. München.

Nachtwey, Oliver (2016): Abstiegsgesellschaft. Über das Aufbegehren in der regressiven Moderne. Berlin.

Pfeiffer, Sabine (2010): Technisierung von Arbeit. In: Böhle, Fritz; Voß, Günter F.; Wachtler, Günther (Hg.): Handbuch Arbeitssoziologie. Wiesbaden: 231-261.

Srnicek, Nick/Williams, Alex (2015): Inventing the Future. Postcapitalism and a World without Work. London-New York.

Urban, Hans-Jürgen (2016): Arbeiten in der Wirtschaft 4.0. Über kapitalistische Rationalisierung und digitale Humanisierung. In: Schröder, Lothar/Urban, HansJürgen (Hg.): Gute Arbeit 2016. Digitale Arbeitswelt - Trends und Anforderungen. Frankfurt/M: 21-45. 


\section{Der PROKLA Förderverein}

Die PROKLA erscheint seit 1971 und bietet politisch engagierte sozialwissenschaftliche und ökonomische Analysen. Allein von den Verkaufserlösen kann sich die PROKLA nicht finanzieren und in die Abhängigkeit von Parteien oder großen Verlagen wollte sie sich nie begeben. Deshalb wird die PROKLA von einem Förderverein herausgegeben, der „Vereinigung zur Kritik der politischen Ökonomie e.V.“, die jährlich in ihrer Vollversammlung die Redaktion der Zeitschrift wählt und die nächsten Themenschwerpunkte diskutiert.

Kritische Sozialwissenschaft kann nicht dem Markt überlassen werden. Ohne solidarische Strukturen und finanzielle Unterstützung sind Zeitschriften wie die PROKLA kaum möglich. Die regelmäßigen finanziellen Beiträge der Vereinsmitglieder ermöglichen das Erscheinen der PROKLA, sie schaffen die Voraussetzungen für Kontinuität und Planbarkeit, wie sie für die Redaktionsarbeit unabdingbar sind. Wir freuen uns über weitere Mitglieder, regelmäßige Spenden oder einmalige Zuwendungen. Weitere Informationen teilen wir gerne per E-Mail mit (redaktion@prokla.de).

Vereinigung zur Kritik der politischen Ökonomie e.V.

IBAN: DE17 100100100538135100 - BIC: PBNKDEFF

Postbank Berlin

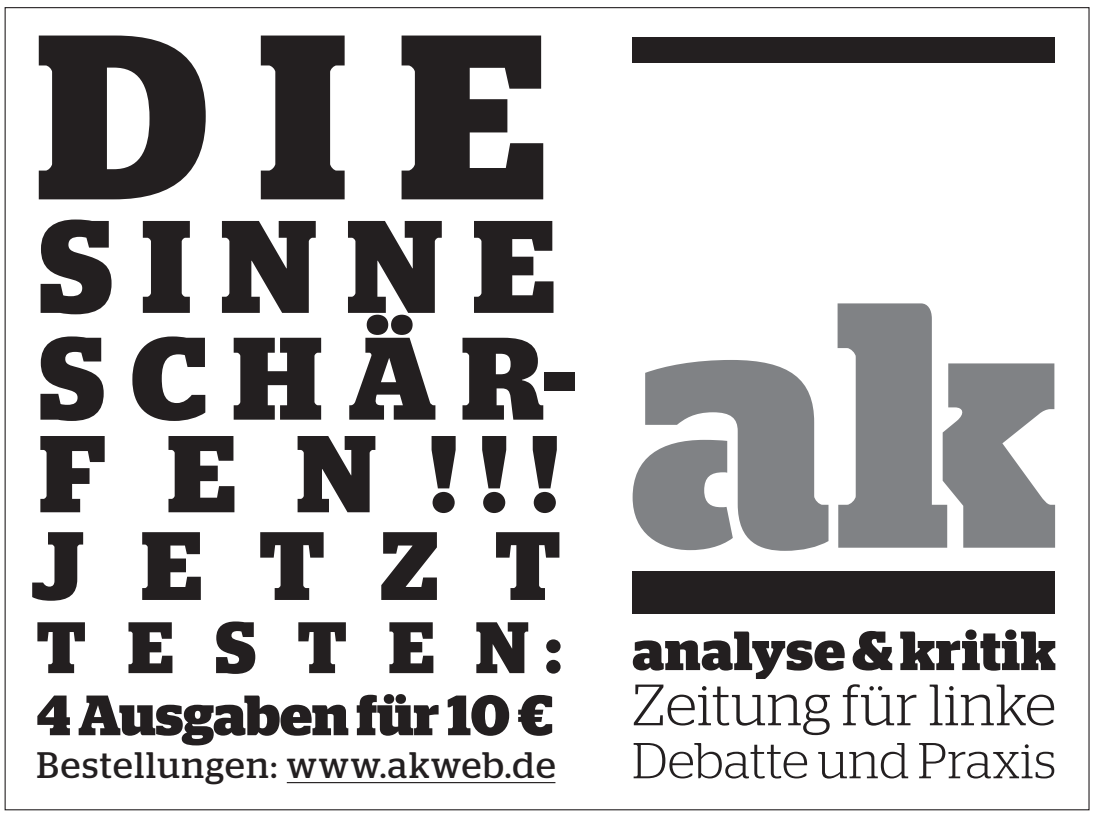




\section{PROKLA 188: Gesellschaftskritik \& 150 Jahre Kritik der politischen Ökonomie}

Seit einigen Jahren hat Gesellschaftskritik Hochkonjunktur - und mit ihr die disziplinäre Selbstverständigung darüber, welche Möglichkeiten und Aufgaben einer „kritischen“ Soziologie zukommen. Zahlreiche Beiträge verlieren dabei aber zunehmend die Kritik der gesellschaftlichen Verhältnisse zugunsten umfassender selbstreflexiver Verständigungen aus dem Blick. Kritik erstarrt allzu häufig zur akademischen Geste. Das geplante Heft liefert eine kritische Revision der vielgestaltigen Kritik-Konjunktur, wobei es mitunter um die universitären Produktionsbedingungen von Kritik geht und die unterschiedlichen Kritikparadigmen und die ihnen eigenen Tendenzen zur Post-Kritik. Das erklärte Ziel des Heftes ist es, die Fallstricke und das gesellschaftskritische Potenzial der Ansätze mit Blick auf das gegenwärtige Erstarken rechter und regressiver Kräfte auszuloten.

Für Gesellschaftskritik ist Karl Marx zentraler Bezugspunkt. Vor bald 150 Jahren erschien erstmals Das Kapital, was Anlass ist, seine einflussreichste ökonomiekritischen Schriften zu würdigen. Es ist für eine radikale Gesellschaftskritik wichtig, sich das kritische Potenzial zu vergegenwärtigen und sie zu aktualisieren, selbstkritisch Gewissheiten und zweifelhafte Traditionslinien zu überprüfen und zu hinterfragen.

\section{PROKLA 189: Autoritärer Populismus \& Kämpfe um die globale Inwertsetzung von Land und Rohstoffen}

Das Jahr 2017 wird von einer Reihe von Wahlen bestimmt sein. In vielen Ländern existieren starke autoritär-populistische Parteien. Einerseits sind diese für einen starken Nationalstaat, andererseits verfolgen sie in bestimmten Bereichen das neoliberale Ziel des Abbaus des Staates. Das Heft widmet sich den politischen Zielen der autoritär-populistischen Parteien, den Wahlen und den politischen Konstellationen. Dies schließt die programmatischen Diskussionen, das Wahlverhalten und die Unterstützung durch verschiedene Teile der Bevölkerung mit ein.

Der zweite Heftschwerpunkt diskutiert die aktuellen, zum Teil aggressiven Bemühungen um die Sicherung des Zugriffs auf Rohstoffe und Land, die neue Konflikte und Kämpfe ausgelöst und alte verschärft haben. Diese Entwicklung geht mit negativen ökologischen und sozialen Folgen einher. Aktuelle Inwertsetzungsprozesse von Land und Rohstoffen sind häufig Teil nationaler und internationaler Entwicklungsstrategien. Weltweit leisten Bauern und Bäuerinnen, AnwohnerInnen und AktivistInnen und schließlich auch Staaten jedoch Widerstand gegen die Ausweitung der s „extraktiven Grenzen“. Diese Kämpfe sind oft nicht nur lokal verankert, sondern über Regionen, Länder und Kontinente hinweg organisiert. 